\title{
MOBILIZAÇÃO PRECOCE EM CRIANÇAS CRITICAMENTE DOENTES: UMA REVISÃO INTEGRATIVA
}

\author{
Early Mobilization in critically ill children: a integrative review
}

\section{Susana Lígia da Silva Rodrigues"}

${ }^{1}$ Fisioterapeuta. Especialista em Saúde da Criança e Adolescente. Hospital Universitário Lauro Wanderley/Paraíba. Email: susanaligia@hotmail.com.

\section{Bárbara Carla de Araújo Rodrigues $^{2}$}

${ }^{2}$ Fisioterapeuta. Especialista em Saúde da Criança e Adolescente. Hospital Universitário Lauro Wanderley / Paraíba. Email: barbara_baaby@hotmail.com.

\section{Eliza Juliana da Costa Eulálio $^{3}$}

${ }^{3}$ Fisioterapeuta. Doutora em Engenharia de Processos. Coordenadora Técnica de Fisioterapia da Unidade Neonatal e Pediátrica. Hospital Universitário Lauro Wanderley/Paraíba.

Email: elizafisioterapia@hotmail.com.

\section{Renata Cavalcanti Farias ${ }^{4}$}

${ }^{4}$ Fisioterapeuta. Mestre em Terapia Intensiva. Coordenadora Técnica de Fisioterapia da UTI Pediátrica. Hospital Universitário Lauro Wanderley / Paraíba.

Email: renatacfarias@hotmail.com.

\section{Andressa Bomfim Lugon Favero $^{5}$}

${ }^{5}$ Fisioterapeuta. Mestre em Ciências Fisiológicas. Coordenadora Técnica de Fisioterapia da Clínica Médica. Hospital Universitário Lauro Wanderley/Paraíba. Email: lugonandressa@hotmail.com.

\begin{abstract}
RESUMO
A mobilização precoce vem se destacando na última década por ser uma estratégia alternativa para minimizar os efeitos deletérios do imobilismo no leito no paciente crítico. Consiste de atividades terapêuticas progressivas que se iniciam logo após a estabilização das alterações fisiológicas do paciente e oferece benefícios a curto, médio e longo prazo. Vários trabalhos evidenciam seus efeitos na população adulta, entretanto, pesquisas feitas com crianças são escassas. Objetivo: Verificar e reunir as evidências científicas disponíveis na literatura sobre mobilização precoce em crianças criticamente doentes. Metodologia: Trata-se de uma revisão sistemática de literatura do tipo integrativa, por meio da busca de artigos científicos nas bases de dados SciELO, BVS e PubMed, utilizando os descritores "children", "critical ill", "early mobilization", "pediatrics" e "rehabilitation", nos idiomas português e inglês, com textos completos publicados entre os anos de 2013 e 2018. Resultados: Foram encontrados 183 artigos elegíveis, dos quais 9 foram selecionados para serem revisados. Os trabalhos abordaram, principalmente, as barreiras, os benefícios, a segurança e a viabilidade da mobilização precoce nas Unidades de Terapia Intensiva Pediátricas (UTIPs), bem como o envolvimento do fisioterapeuta na reabilitação precoce da criança criticamente doente. Conclusões: A mobilização precoce é segura, viável, oferece poucos riscos ao paciente, favorece o aumento da mobilidade das crianças e pressupõe um menor tempo de internação. $\mathrm{O}$ fortalecimento da equipe multidisciplinar pode contribuir para a implementação da mobilização precoce em UTIPs.
\end{abstract}

PALAVRAS-CHAVE: Criança; Doença Crítica; Mobilização Precoce; Reabilitação. 


\section{ABSTRACT}

Early mobilization has been highlighted in the last decade as an alternative strategy to minimize the deleterious effects of bed rest in critical patients. It consists of progressive therapeutic activities that begin soon after stabilizing the physiological changes of the patient and offers benefits in the short, medium and long term. Several studies show its effects on the adult population, however, research on children is scarce. Objective: To verify and gather the scientific evidence available in the literature on early mobilization in critically ill children. Methodology: This is a systematic review of literature of the integrative type, through the search of scientific articles in the SciELO, BVS and PubMed, using the descriptors "children", "critical ill", "early mobilization", "pediatrics" and "rehabilitation", in Portuguese and English, with full texts published between 2013 and 2018. Results: 183 eligible articles, of which 9 were selected for review. The work focused on the barriers, benefits, safety and feasibility of early mobilization in Pediatric Intensive Care Units (PICUs), as well as the involvement of the physiotherapist in the early rehabilitation of critically ill children. Conclusions: Early mobilization is safe, viable, offers few risks to the patient, favors the increase of children's mobility and presupposes a shorter hospitalization time. The strengthening of the multidisciplinary team can contribute to the implementation of early mobilization in PICUs.

KEYWORDS: Child; Critical IIIness; Early Mobilization; Rehabilitation.

\section{INTRODUÇÃO}

Conforme Pais (2012), doente crítico é aquele que necessita de monitorização avançada e intervenção terapêutica devido à falência de um ou mais órgãos ou sistemas. O Ministério da Saúde define paciente crítico como "aquele que se encontra em risco iminente de perder a vida ou função de órgão/sistema do corpo humano, bem como aquele em frágil condição clínica" (BRASIL, 2011).

Durante muito tempo o repouso absoluto foi preconizado como conduta eficaz para recuperação dos doentes críticos. Atualmente, sabe-se que imobilidade é um problema grave e muito frequente, afetando cerca de $60 \%$ dos adultos e deixando sequelas que podem durar anos após a alta hospitalar (PERME, CHANDRASHEKAR, 2009).

O repouso prolongado, quando associado à ventilação mecânica invasiva (VMI), leva à perda de fibras musculares gerando fraqueza da musculatura respiratória e periférica, frequentemente denominada de fraqueza adquirida na Unidade de Terapia Intensiva (FAUTI). Estudos mostram que são necessários somente sete dias para que a força muscular diminua em $30 \%$, com perda adicional de $20 \%$ a cada semana (FRANÇA et al., 2012; GODOY et al., 2015).

O longo período de internação e restrição ao leito também pode gerar problemas negativos às crianças, tais como: fraqueza, distúrbios emocionais, comportamentais, cognitivos e funcionais, afetando tanto sua qualidade de vida como a de seus cuidadores. É importante ressaltar que crianças com condições crônicas e deficiências representam um aumento da proporção de admissões nas Unidades de Terapia Intensiva Pediátricas (UTIPs), perpassando por longos períodos de internação, ocasionando implicações importantes para sua recuperação, além de elevar os custos hospitalares (CHOONG et al., 2014). 
Cada vez mais têm sido estudadas novas tecnologias e alternativas para minimizar os reconhecidos efeitos nocivos do repouso prolongado. A reabilitação logo no início da doença crítica pode influenciar positivamente na capacidade de recuperação da criança. Nesse sentido, a Mobilização Precoce (MP) destaca-se por se tratar de atividades de mobilização que se iniciam logo após a estabilização das alterações fisiológicas do paciente, por meio de atividades terapêuticas progressivas que vão desde exercícios motores no leito até a deambulação (MOTA; SILVA, 2012).

Muitos desses estudos são feitos com adultos e indicam seus benefícios a curto, médio e longo prazo, porém sua prática em crianças ainda representa um desafio. Atualmente, a fisioterapia compõe a equipe multidisciplinar, sendo o fisioterapeuta o profissional responsável por realizar exercícios terapêuticos, inclusive de mobilização precoce, a fim de prevenir e tratar comorbidades (SARTI; VECINA; FERREIRA, 2016).

Estudos sobre mobilização precoce em crianças em condições críticas ainda são incipientes e pouco abordados em pesquisas científicas, carecendo de diretrizes e protocolos validados voltados à sua prática. Sendo assim, este trabalho teve como objetivo principal verificar e reunir evidências atuais sobre mobilização precoce em crianças criticamente doentes.

\section{MATERIAL E MÉTODOS}

Trata-se de uma revisão sistemática do tipo integrativa que primariamente estabeleceu a seguinte questão norteadora: Quais as evidências atuais sobre mobilização precoce em crianças criticamente doentes? A segunda etapa envolveu a busca nas bases de dados SciELO (Scientific Electronic Library Online), BVS (Biblioteca Virtual em Saúde) e PubMed (Biblioteca Nacional de Medicina dos Estados Unidos), cruzando-se os seguintes descritores e palavras-chave: "children", "critical ill", "early mobilization”," pediatrics" e "rehabilitation".

Os critérios de inclusão definidos para a seleção dos trabalhos foram: artigos originais online que abordem a mobilização precoce em crianças de 0 a 18 anos, gratuitos e disponíveis na íntegra, na literatura nacional e internacional (português e inglês), publicados entre 2013 e 2018, considerando que esta é uma prática recente na população pediátrica. Após a leitura do título, resumo e texto na íntegra, foram excluídos aqueles que não tinham correlação com o objetivo proposto, bem como estudos de revisão e editoriais. A pesquisa bibliográfica foi realizada no período de novembro de 2017 a fevereiro de 2018.

\section{RESULTADOS E DISCUSSÃO}

Foram encontrados, ao todo, 183 (cento e oitenta e três) artigos com os descritores utilizados e, após a análise dos critérios de inclusão e exclusão, apenas 9 (nove) foram revisados (Figura 1). Os artigos constaram de ensaios clínicos, que avaliaram a viabilidade e a segurança de protocolos de mobilização precoce, e estudos transversais e retrospectivos, que buscaram identificar as percepções da equipe multidisciplinar e os fatores preditivos e limitantes à implementação de programas de mobilização precoce nas UTIPs (Quadro 1). 
Figura 1. Fluxograma de amostra de artigos selecionados para revisão.

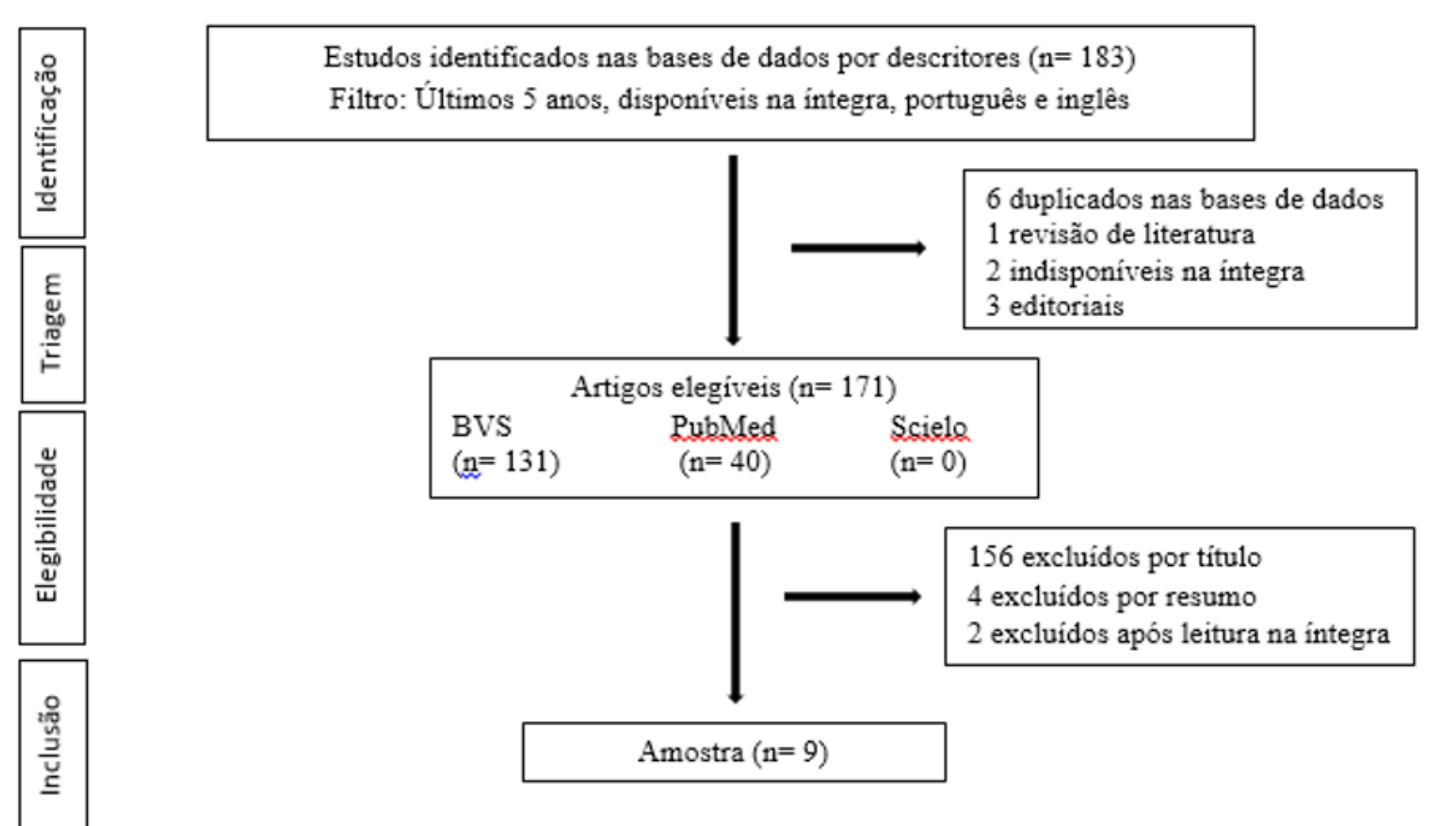

Fonte: Dados da pesquisa, 2018. 
Quadro 1. Visão geral dos estudos relacionados à Mobilização Precoce em crianças criticamente doentes, João Pessoa - PB, Brasil, 2018 (continua).

\begin{tabular}{|c|c|c|c|c|c|}
\hline $\begin{array}{l}\text { Autor, Ano e } \\
\text { Local }\end{array}$ & Tipo de estudo & Objetivos & $\begin{array}{c}\text { Amostra, Idade e } \\
\text { Tempo de internaçäo }\end{array}$ & Intervenções & Principais Resultados \\
\hline $\begin{array}{l}\text { Coşkun-Benlidayı } \\
\text { et al. } \\
\text { (2015) } \\
\text { Turquia }\end{array}$ & Relato de Caso & $\begin{array}{l}\text { Apresentar segurança da } \\
\text { MP e EENM na FAUTI } \\
\text { com dificuldade de } \\
\text { desmame da VMI. }\end{array}$ & $\begin{array}{c}n=1 \\
12 \text { anos } \\
44^{\circ} \text { dia }\end{array}$ & $\begin{array}{c}\text { UTIP: MP } \\
\text { UI: fortalecimento + EENM }\end{array}$ & $\begin{array}{l}\text { Melhora na cognição, mobilidade e } \\
\text { AVD's; } \\
\text { MP e EENM seguras e eficazes na } \\
\text { FAUTI pediátrica. }\end{array}$ \\
\hline $\begin{array}{l}\text { Wieczorek et al. } \\
\text { (2016) } \\
\text { EUA }\end{array}$ & Observacional & $\begin{array}{l}\text { Determinar segurança e } \\
\text { viabilidade de um } \\
\text { programa de MP. }\end{array}$ & $\begin{array}{l}\mathrm{n}=200 \\
1-17 \text { anos } \\
\geq 3 \text { dias }\end{array}$ & $\begin{array}{c}3 \text { níveis de atividade } \\
\text { G1: Não mobilidade (leito) } \\
\text { G2: Mobilização } \\
\text { Questionário pós-intervenção }\end{array}$ & $\begin{array}{l}\text { Maior Participação do FT e T.O., } \\
\text { aumento e início mais precoce da } \\
\text { mobilização; } \\
\text { Nenhum evento adverso; } \\
\text { MP segura e viável. }\end{array}$ \\
\hline $\begin{array}{l}\text { Choong et al. } \\
\text { (2017) } \\
\text { Canadá }\end{array}$ & $\begin{array}{l}\text { Ensaio Clínico } \\
\text { Piloto } \\
\text { Randomizado } \\
\text { Controlado }\end{array}$ & $\begin{array}{l}\text { Avaliar viabilidade e } \\
\text { eficácia da MP usando } \\
\text { ciclismo no leito como } \\
\text { complemento da } \\
\text { fisioterapia. }\end{array}$ & $\begin{array}{c}\mathrm{n}=30 \\
(\mathrm{G} 1: 20 \text { e } \mathrm{G} 2: 10) 3-17 \\
\text { anos } \\
\geq 48 \mathrm{~h}\end{array}$ & $\begin{array}{l}\text { G1: Fisioterapia convencional } \\
\text { G2: Fisioterapia convencional + } \\
\text { Cicloergômetro em MMII }\end{array}$ & $\begin{array}{l}\text { Otimização de tempo e intensidade da } \\
\text { mobilização; } \\
\text { Nenhum evento adverso; } \\
\text { MP segura e viável. }\end{array}$ \\
\hline $\begin{array}{l}\text { Choong et al. } \\
\text { (2013) } \\
\text { Canadá }\end{array}$ & Transversal & $\begin{array}{l}\text { Avaliar conhecimento, } \\
\text { percepções e práticas } \\
\text { declaradas de MP entre } \\
\text { médicos e } \\
\text { fisioterapeutas. }\end{array}$ & $\begin{array}{c}\mathrm{n}=137 \\
102 \text { médicos } \\
35 \text { fisioterapeutas }\end{array}$ & $\begin{array}{c}\text { Entrevista sobre barreiras à MP, } \\
\text { tempo, natureza e limites da } \\
\text { reabilitação e carga de trabalho da } \\
\text { equipe }\end{array}$ & $\begin{array}{c}\text { Falta de diretrizes, ordens médicas, } \\
\text { escassez de recursos, segurança do } \\
\text { paciente; } \\
\text { Predomínio de FR e ADM passiva; } \\
\text { Frequência e duração dependem da } \\
\text { gravidade da doença, sedação, } \\
\text { colaboração do paciente e } \\
\text { disponibilidade de FTs }\end{array}$ \\
\hline $\begin{array}{l}\text { Choong et al. } \\
\text { (2014) } \\
\text { Canadá }\end{array}$ & $\begin{array}{c}\text { Coorte } \\
\text { Retrospectivo }\end{array}$ & $\begin{array}{l}\text { Avaliar as práticas de } \\
\text { reabilitação, frequência e } \\
\text { barreiras à MP. }\end{array}$ & $\begin{array}{c}n=600 \\
3-17 \text { anos } \\
\geq 24 h\end{array}$ & $\begin{array}{c}\text { G1 (não-mobilização): FR, } \\
\text { posicionamento ou alongamento } \\
\text { G2 (mobilização): fortalecimento, } \\
\text { mobilização no leito, } \\
\text { transferências, treino de marcha e } \\
\text { deambulação }\end{array}$ & $\begin{array}{l}\text { MP infrequente, focada na FR; } \\
\text { Falta de diretrizes, ordens médicas, } \\
\text { bloqueio neuromuscular; } \\
\text { Crianças maiores e admissão no } \\
\text { inverno são preditores de MP; } \\
\text { Nenhum evento adverso. }\end{array}$ \\
\hline
\end{tabular}


Quadro 1. Visão geral dos estudos relacionados à Mobilização Precoce em crianças criticamente doentes João Pessoa - PB, Brasil, 2018 (conclusão).

\begin{tabular}{|c|c|c|c|c|c|}
\hline $\begin{array}{l}\text { Autor, Ano e } \\
\text { Local }\end{array}$ & Tipo de estudo & Objetivos & $\begin{array}{l}\text { Amostra, Idade } \\
\text { e Tempo de } \\
\text { internação }\end{array}$ & Intervenções & Principais Resultados \\
\hline $\begin{array}{l}\text { Joyce et al. } \\
\text { (2018) } \\
\text { EUA }\end{array}$ & Transversal & $\begin{array}{l}\text { Avaliar o conhecimento da } \\
\text { equipe multidisciplinar } \\
\text { sobre MP. }\end{array}$ & $\begin{array}{l}\mathrm{n}=71 \\
\text { profissionais }\end{array}$ & $\begin{array}{c}\text { Entrevista sobre percepções da equipe } \\
\text { sobre MP }\end{array}$ & $\begin{array}{c}\text { MP infrequente e em crianças maiores; } \\
\text { Pouca participação do FT. e T.O.; } \\
\text { Riscos relacionados à segurança do } \\
\text { paciente. }\end{array}$ \\
\hline $\begin{array}{l}\text { Johnston et al. } \\
\qquad \begin{array}{l}\text { (2017) } \\
\text { Canadá }\end{array}\end{array}$ & $\begin{array}{l}\text { Retrospectivo } \\
\text { Transversal }\end{array}$ & $\begin{array}{l}\text { Examinar padrões de } \\
\text { referência para } \\
\text { fisioterapia de crianças } \\
\text { admitidas em um centro } \\
\text { de hemato/oncologia; }\end{array}$ & $\begin{array}{l}\mathrm{n}=228 \\
\text { prontuários } \\
4-18 \text { anos } \\
\geq 48 \mathrm{~h}\end{array}$ & $\begin{array}{l}\text { Análise de prontuários com foco em } \\
\text { tempo para início, frequência, } \\
\text { barreiras, natureza e participação do } \\
\text { FT na MP }\end{array}$ & $\begin{array}{c}\text { Mobilização até o } 2^{\circ} \text { dia de admissão, } 4 \\
\text { dias/semana; } \\
\text { Predomínio de ortostatismo e deambulação; } \\
\text { MP: cuidadores, enfermeiros e } \\
\text { fisioterapeutas; } \\
\text { Barreira: dor. }\end{array}$ \\
\hline $\begin{array}{l}\text { Miura et al. } \\
\text { (2018) } \\
\text { EUA }\end{array}$ & $\begin{array}{l}\text { Retrospectivo } \\
\text { Pré-intervenção }\end{array}$ & $\begin{array}{l}\text { Identificar fatores } \\
\text { preditivos para o início da } \\
\text { reabilitação precoce. }\end{array}$ & $\begin{array}{l}n=100 \\
1-17 \text { anos } \\
\geq 3 \text { dias }\end{array}$ & $\begin{array}{l}\text { Análise de dados de um programa de } \\
\text { MP avaliando tempo para início, } \\
\text { consulta do FT e dados da função } \\
\text { basal do paciente }\end{array}$ & $\begin{array}{l}\text { Função basal anormal e maior gravidade da } \\
\text { doença são preditores de MP com FT. }\end{array}$ \\
\hline $\begin{array}{l}\text { Hollander et } \\
\text { al. (2014) } \\
\text { Canadá }\end{array}$ & Retrospectivo & $\begin{array}{l}\text { Determinar a segurança e } \\
\text { viabilidade de um } \\
\text { programa de MP após } \\
\text { colocação de DAV. }\end{array}$ & $\begin{array}{l}\mathrm{n}=17 \\
0-12 \text { anos } \\
\sim 7 \text { dias }\end{array}$ & $\begin{array}{l}\text { Análise de dados de um programa de } \\
\text { MP após colocação de DAV } \\
\text { Crianças < } 1 \text { ano: atividades adaptadas } \\
\text { com participação dos pais } \\
\text { Crianças > 1ano: Atividades p/ crianças }\end{array}$ & $\begin{array}{c}3 \text { crianças }<1 \text { ano não atingiram todos os } \\
\text { objetivos }(5 / 8) \\
\text { Nenhum evento adverso; } \\
\text { MP segura e viável. }\end{array}$ \\
\hline
\end{tabular}

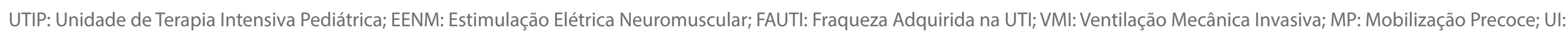

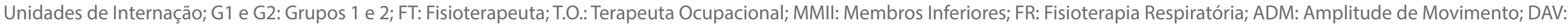
Dispositivo de Assistência Ventricular. 


\section{Mobilização precoce}

De acordo com Mota e Silva (2012), as atividades de mobilização precoce objetivam aumentar a força muscular, funcionalidade, redução do tempo de internação e de custos e aumento da sobrevida pós-alta hospitalar. Houve semelhança nas propostas de tratamento encontradas, iniciando com a mobilização no leito, progredindo para sedestação à beira do leito, ortostatismo, sedestação na cadeira e deambulação.

As recomendações da força tarefa da European Respiratory Society and European Society of Intensive Care Medicine, voltadas ao doente crítico adulto, estabelecem que a mobilização precoce trata-se de uma hierarquia de atividades de mobilização na UTI baseada em uma sequência de intensidade do exercício: mudança de decúbitos e posicionamento funcional, mobilização passiva, exercícios ativo-assistidos e ativos, uso de cicloergômetro na cama, sentar na borda da cama, ortostatismo, caminhada estática, transferência da cama para poltrona, exercícios na poltrona e caminhada (GOSSELINK et al., 2008).

Dos estudos encontrados, apenas Choong e et al. (2017) fizeram uso do cicloergômetro no leito, sendo considerado um estudo pioneiro desta modalidade. Neste ensaio clínico denominado "WEECYCLE", os pacientes foram randomizados em dois grupos. O grupo controle recebeu apenas fisioterapia convencional e o grupo ciclismo, fisioterapia convencional e cicloergômetro (versão pediátrica) em MMIl durante 30 minutos, 5 dias por semana. As crianças foram monitoradas constantemente e não houve intercorrências. Como resultado, demonstraram que o ciclismo leito pode facilitar a mobilização, seja de forma passiva ou ativa, otimizando o tempo e intensidade da fisioterapia, além de mostrar-se uma terapia segura e viável.

O estudo de Hollander et al. (2014) investigou a viabilidade de um programa de MP em crianças após colocação de dispositivo de assistência ventricular (DAV). O programa incluía atividades progressivas desde o leito até a deambulação, realização de atividades da vida diária (AVDs) e jogos, adequados por idade. Como resultado, observou-se que de 17 crianças participantes, 15 foram capazes de realizar todas as etapas até o momento do transplante de coração e aqueles que não conseguiram não o fizeram por fatores não relacionados à terapia, reforçando a viabilidade e segurança da MP em crianças criticamente doentes.

É importante destacar que, em relação aos demais estudos encontrados, neste houve a preocupação de elaborar atividades adaptadas à faixa etária das crianças $(<1$ ano e > 1 ano), considerando-se a heterogeneidade das fases de desenvolvimento na população pediátrica. Além disso, incluiu a participação dos pais neste processo como corresponsáveis pela recuperação de seus filhos, revelando-se uma terapia segura e viável também em crianças menores. Jonston et al. (2017) demonstraram que o envolvimento dos pais na terapia diminui o estresse emocional, aumenta sensação de segurança e diminui a ansiedade das crianças.

Wieczorek et al. (2016) apresentaram um programa multicomponente e interdisciplinar para promover a MP de crianças criticamente doentes, baseado em 3 níveis, de acordo com os parâmetros fisiológicos, clínicos e nível de desenvolvimento da criança. As atividades foram divididas em atividades de não mobilidade (mobilização passiva, ADM ativa, posicionamento ativo ou passivo no leito e colocação de órtese) e atividades de mobilidade (sentar à beira do leito, sentar-se na cadeira, ficar em pé, transferências, deambulação e jogos). Como resultado, houve aumento e início mais precoce da mobilização, maior envolvimento da Fisioterapia e Terapia Ocupacional (T.O.) e ausência de efeitos adversos relacionados à terapia. A abordagem multidisci- 
plinar mostrou-se um componente importante e facilitador na implementação da MP. Houve ainda um relato de caso, feito por Coşkun-Benlidayı et al. (2015), de uma criança de 12 anos, diagnosticada com glomerulonefrite membranoproliferativa, que desenvolveu FAUTI e dificuldade de desmame da VMI. A mobilização ocorreu no $44^{\circ}$ dia de internação da UTIP, após acionamento da equipe de reabilitação, sendo este considerado o momento em que a criança estava apta para receber mobilização pela equipe. As atividades iniciaram com mobilização ativo-assistida no leito, progredindo para rolar, supino-sentado, sentar sem apoio na borda da cama e deambulação com apoio. Após esta fase, a criança recebeu alta da UTIP e foi encaminhada para unidade de internação onde foi dado prosseguimento das condutas.

Observa-se que a MP é uma fase imprescindível da reabilitação da criança criticamente doente, servindo de base preparatória para novas etapas da reabilitação, à medida que melhora o condicionamento físico da criança, evitando comorbidades e aumento da sobrevida após alta hospitalar.

\section{Frequência e Tempo para início}

Choong et al. (2014) definiram "reabilitação" como o tratamento que visa facilitar a recuperação do paciente, seja por lesão ou doença, cujos profissionais que se enquadram nesta categoria são Fisioterapeuta, Terapeuta Ocupacional e Fonoaudiólogo e definiram "mobilização precoce" como qualquer terapia que utilize da mobilização no período dentro das primeiras 48h de admissão. Já Wieczorek et al. (2016), estabelece que as atividades devem se iniciar nos primeiros 3 dias da admissão e destinam-se a manter ou restaurar a força e a função do paciente. Alguns estudos utilizaram ainda, o termo reabilitação precoce, denotando, no geral, as atividades de mobilização iniciadas precocemente na reabilitação da criança criticamente doente.

O estudo de Miura et al. (2018) mostrou que o comprometimento motor basal, a capacidade cognitiva, ventilação mecânica e quantidade de dispositivos de uso contínuo foram associados à mobilização precoce, enquanto que admissão em dias próximos ao fim de semana foi associada à mobilização tardia. Outro estudo, Choong et al. (2014), apontou o bloqueio neuromuscular como fator de adiamento da mobilização e admissão no inverno, infusão de sedativos, associaram-se à mobilização precoce. Miura et al. (2018) e Choong et al. (2013) afirmam que a MP é mais direcionada às crianças mais graves, pois há déficit de fisioterapeutas nos ambientes onde as pesquisas foram desenvolvidas e estas crianças são vistas, portanto, como prioridade.

Em um estudo multicêntrico, Choong et al. (2014) avaliaram as práticas de reabilitação nas UTIPs do Canadá ( $\mathrm{n}=600$ crianças), constataram que apenas metade das crianças recebeu reabilitação, esta era focada na parte respiratória e apenas 9,7\% destas recebeu mobilização precoce. Joyce et al. (2018) revelaram que a maior parte dos pacientes nunca recebeu mobilização precoce. Curiosamente, Choong et al. (2017) no seu ensaio clínico com cicloergômetro verificaram que, com o incremento do ciclismo, mais crianças eram mobilizadas, porém, para realizar esta atividade, exigia-se dos pacientes um maior entendimento e capacidade de compreensão beneficiando sobremaneira as crianças maiores, segregando as menores e os bebês.

\section{Barreiras, benefícios e participação do fisioterapeuta na mobilização precoce}

Houve similaridade entre os estudos quanto às barreiras e limitações que difi- 
cultam a prática da mobilização precoce. As barreiras por parte dos profissionais foram: falta de diretrizes pediátricas, necessidade de prescrição médica para reabilitação, escassez de recursos, aumento da carga de trabalho, déficit de fisioterapeutas nos hospitais em finais de semana. Outras limitações estavam ligadas à segurança do paciente e incluíram, sobretudo, dor, nível de sedação, possibilidade de intercorrências, risco de perda de acesso venoso central, risco de desconexão de dispositivos de uso contínuo e tubo endotraqueal (CHOONG et al., 2013; CHOONG et al., 2014; JOHNSTON et al., 2017; JOYCE et al., 2018).

Os benefícios da MP ainda não foram bem elucidados por ser uma prática relativamente nova em crianças. Joyce et al. (2017) apontaram a redução do tempo de internação, do delirium e custos hospitalares como fatores favoráveis. Os benefícios nessa população estão relacionados principalmente à segurança e viabilidade, cabendo ressaltar a escassez de estudos com rigor metodológico que comprovem a sua eficácia. Contudo, nota-se o aumento da mobilidade das crianças, melhora da cognição e pressupõe uma antecipação da alta hospitalar.

No estudo de Choong et al. (2013), a maioria dos profissionais entrevistados foi de médicos, enfermeiras e fisioterapeutas. Quando indagados sobre seu conhecimento e práticas de MP, declararam sentirem-se bem treinados para sua prática. Porém, neste estudo e tal como ocorreu no estudo de Joyce et al. (2018), o fisioterapeuta era pouco envolvido na recuperação das crianças criticamente doentes. Este fato também foi apontado por Johnston et al. (2017), onde a mobilização das crianças ficava a cargo da enfermagem e dos cuidadores e restrito à escala dos fisioterapeutas.

Este achado pode ser justificado, conforme Choong et al. (2013) explicam, pelo fato de que nas instituições canadenses possuem recursos fisioterapêuticos limitados e que os fisioterapeutas não estão disponíveis $24 \mathrm{~h}$ nas UTIP. Constataram que apenas metade das UTIPs canadenses tem acesso à reabilitação, pois os profissionais trabalham em outros setores do hospital e participam dos cuidados intensivos na UTIP somente quando são solicitados. Miura et al. (2018) complementam que os pedidos de fisioterapia e terapia ocupacional são solicitados simultaneamente.

Cabe ressaltar ainda que nestes países, conforme Choong et al. (2013) e Choong et al. (2014), mais da metade dos entrevistados sentiram que os médicos não percebiam MP como uma prioridade de atendimento ao paciente e que estes não são os primeiros a responsáveis pela identificação da necessidade de mobilização precoce, reconhecendo o fisioterapeuta como o profissional adequado para a função.

O pouco envolvimento, encontrado nos estudos, por parte destes profissionais no tratamento da doença crítica das crianças esteve também intimamente ligado à necessidade de prescrição médica para sua prática. Contudo, a força tarefa da European Respiratory Society and European Society of Intensive Care Medicine recomenda que o fisioterapeuta deve ser o profissional responsável pela implantação e gerenciamento do plano de mobilização (GOSSELINK et al., 2008).

No Brasil, a legislação vigente indica que é competência do Fisioterapeuta “(...) prescrever, baseado no constatado na avaliação físico-funcional as técnicas próprias da Fisioterapia, qualificando-as e quantificando-as; dar ordenação ao processo terapêutico baseando-se nas técnicas fisioterapêuticas indicadas (...)", dentre outras funções. Neste sentido, evidencia-se que o fisioterapeuta não deve ser conduzido por diagnósticos médicos nem haver subordinação desta profissão em relação a outras (BRASIL, 1987). O desafio da implementação da MP demanda uma mudança cultura dos hospitais, sensibilização e estimulação de todos os envolvidos no cuidado da criança criticamente doente. Nesta perspectiva, um passo importante é estabelecer o fortalecimento da 
equipe multidisciplinar, melhorando a comunicação entre os profissionais sobre indicações, contraindicações e custo-benefício da MP, visando um objetivo comum que é a recuperação efetiva do paciente.

\section{Segurança e viabilidade}

O estudo retrospectivo de coorte feito por Choong et al. (2014) comparou um grupo que recebeu terapia de mobilização: fortalecimento, mobilização no leito, transferências, treino de marcha e deambulação com um grupo que não recebeu mobilização: apenas FR e posicionamento ou alongamento. Eventos adversos como instabilidade hemodinâmica, descompensação respiratória, deslocamentos de tubo e intolerância ao exercício foram analisados e não representaram diferenças estatisticamente significativas entre os grupos intervenção e controle. Também foi constatado que os profissionais se sentiam mais seguros em realizar a MP em crianças maiores, tal como foi observado no estudo de Joyce et al. (2017).

Um achado importante desta revisão é que todos os estudos tiveram como cenário as unidades de cuidado intensivo. É importante destacar que a mobilização precoce não se restringe somente às UTIs. A criança criticamente doente pode estar hospitalizada nas unidades de internação, passando por longos períodos de repouso, restritas ao leito e também está sujeita ao desenvolvimento de comorbidades, perdas funcionais e cognitivas, distúrbios psicológicos, além de gerar custos maiores ao hospital devido internação prolongada.

Um estudo recente feito em larga escala ( $n=12.490)$ por Liu et al. (2017), intitulado "MOVE ON", objetivou implementar e avaliar uma estratégia para promover a mobilização precoce de pacientes idosos hospitalizados em unidades de internação em 14 hospitais de Ontário/Canadá. Os critérios definiam que os pacientes deveriam ser avaliados quanto à mobilidade dentro das primeiras $24 \mathrm{~h}$ de internação, a mobilização deveria ocorrer 3 vezes ao dia, ser progressiva e adaptada às capacidades de cada paciente. Como desfecho, mais pacientes saíram do leito mais vezes por dia $(p<0.001)$ e o tempo de internação reduziu em 6.1 dias em comparação com o período pré-intervenção.

Embora não seja uma medida de resultados em crianças, este estudo pode fornecer norteamento para novas pesquisas além da UTIP, uma vez que os estudos sobre mobilização precoce em crianças criticamente doentes estão sendo desenvolvidos com base nos resultados satisfatórios obtidos com adultos.

\section{CONSIDERAÇÕES FINAIS}

Este trabalho mostrou que a heterogeneidade dos pacientes se traduz na dificuldade de implementar protocolos seguros e viáveis nesta população. Mobilizar precocemente crianças criticamente doentes requer do fisioterapeuta, além de precauções com a segurança do paciente, estratégias para adesão das crianças à terapia, tanto as menores quanto as maiores.

O incremento do cicloergômetro e do lúdico podem constituir uma ferramenta fundamental neste processo, por meio de atividades adaptadas por faixa etária, conforme o nível de entendimento do paciente. A participação dos pais nas atividades junto ao fisioterapeuta também é importante para aumentar o nível de confiança e facilitar o vínculo terapeuta-paciente, sobretudo nas crianças menores que demandam 
mais atenção.

Vale salientar que as pesquisas foram realizadas em outros países, cuja realidade dos hospitais difere da realidade brasileira, onde os fisioterapeutas estão alocados em outros setores dos hospitais e sua participação na mobilização precoce dos pacientes ocorre quando estes são solicitados. Deste modo, a implantação e fortalecimento da equipe multidisciplinar nos centros de terapia intensiva pode contribuir de forma positiva para a implementação da mobilização precoce nestes ambientes.

Embora vários riscos prováveis tenham sido apontados, nenhum dano ou evento adverso ao paciente decorrente da sua prática foi relatado. Com base na literatura, é inconteste que a MP é segura, viável e traz resultados satisfatórios, oferecendo poucos riscos ao paciente, favorece aumento da mobilidade das crianças e pressupõe um menor tempo de internação que, em longo prazo, pode reduzir custos hospitalares. Observou-se ainda, que os trabalhos desenvolvidos com MP em pediatria são baseados em estudos realizados com pacientes adultos e todos foram realizados em UTIPs. No entanto, as evidências comprovam que a MP deve, também, abranger as unidades de internação. São necessárias mais pesquisas intervencionistas brasileiras, com rigor metodológico para melhor compreensão da MP em crianças, sendo uma lacuna a ser preenchida pela escassez de literatura nessa área do conhecimento.

Espera-se, com este trabalho, fornecer subsídios que contribuam para aprimorar a prática clínica dos profissionais da equipe multidisciplinar, sobretudo fisioterapeutas, facilitar o processo de tomada de decisões baseadas em evidências e colocar em prática a MP como peça indispensável no cuidado do paciente pediátrico criticamente doente.

\section{REFERÊNCIAS}

BRASIL. Ministério da Saúde. Gabinete do Ministro. Portaria n 2.338/2011, de 3 de outubro de 2011. Estabelece diretrizes e cria mecanismos para a implantação do componente Sala de Estabilização (SE) da Rede de Atenção às Urgências. Diário Oficial da União, Brasília, DF, 2011.

BRASIL. Ministério do Trabalho. Conselho Federal de Fisioterapia e Terapia Ocupacional. Resolução no. 80, de 9 de maio de 1987. Diário Oficial da União. Brasília, 21 mai. 1987. n. 93, Seção 1, p. 7609.

CHOONG, K. et al. Acute Rehabilitation Practices in Critically III Children: A Multicenter Study. Pediatric Critical Care Medicine, Mount Prospect, v. 15, n. 6, p. e270-e279, 2014. Disponível em: < https://www.ncbi.nlm.nih.gov/pubmed/24777303>. Acesso em: 24 jan. 2018.

CHOONG, K. et al. Early Exercise in Critically III Youth and Children, a Preliminary Evaluation: The wEECYCLE Pilot Trial. Pediatric Critical Care Medicine, Mount Prospect, v. 18, n, 11, p. e546-e554, 2017. Disponível em: < https://www.ncbi.nlm.nih.gov/ pubmed/28922268>. Acesso em: 20 jan. 2018.

CHOONG, K. et al. Early Mobilization in Critically III Children: A Survey of Canadian Practice. Critical Care Medicine, Mount Prospect, v. 41, n. 7, p. 1745-1753, 2013. Disponível em: <https://www.ncbi.nlm.nih.gov/pubmed/23507722>. Acesso em: 24 jan. 
2018.

COŞKUN-BENLIDAYI, İ. et al. Early rehabilitation of a child with intensive care unit acquired weakness secondary to membranoproliferative glomerulonephritis: A case report. The Turkish Journal of Pediatrics, Ancara, v. 57, n. 4, p. 422-425, 2015. Disponível em: < https://www.ncbi.nlm.nih.gov/pubmed/27186711>. Acesso em: 20 jan, 2018.

FRANÇA, E. É. T. et al. Fisioterapia em pacientes críticos adultos: recomendações do Departamento de Fisioterapia da Associação de Medicina Intensiva Brasileira. Revista Brasileira de Terapia Intensiva, São Paulo, v. 24, n. 1, p. 6-22, 2012.

GODOY, D. A. et al. Intensive Care Unit Acquired Weakness (ICU-AW): a brief and practical review. Reviews in Health Care, Torino, v. 6, n. 1, p. 9-35, 2015. Disponível em: < https://journals.edizioniseed.it/index.php/rhc/article/view/1037>. Acesso em: 15 jan. 2018.

GOSSELINK R. et al. Physiotherapy for adult patients with critical illness: recommendations of the European Respiratory Society and European Society of Intensive Care Medicine Task Force on Physiotherapy for Critically III Patients. Intensive Care Medicine, Paris, v. 34, n. 7, p.1188-1199, 2008. Disponível em: < https://www.ncbi.nlm.nih. gov/pubmed/18283429>. Acesso em: 15 jan, 2018.

HOLLANDER, S. A. et al. An inpatient rehabilitation program utilizing standardized care pathways after paracorporeal ventricular assist device placement in children. The Journal of Heart and Lung Transplantation, Nova lorque, v. 33, n. 6, p. 587-592, 2014. Disponível em: < https://www.ncbi.nlm.nih.gov/pubmed/24468119>. Acesso em: 20 jan. 2018.

JOHNSTON, K,; DELIVA, R.; EVANS, C. Mobilization patterns of children on a hematology/oncology inpatient ward. Pediatric Blood \& Cancer, Medford, v. 64, n. 11, 2017 Disponível em: <https://www.ncbi.nlm.nih.gov/pubmed/28409889>. Acesso em: 20 jan. 2018.

JOYCE, C.L. et al. Provider beliefs regarding early mobilization in the Pediatric Intensive Care Unit. Journal of Pediatric Nursing: Nursing of Childrem and Families, Nova lorque, v.38, p. 15-19, 2018. Disponível em: < https://www.ncbi.nlm.nih.gov/ pubmed/29167075>. Acesso em: 17 jan. 2018.

LIU, B. et al. Outcomes of Mobilisation of Vulnerable Elders in Ontario (MOVE ON): a multisite interrupted time series evaluation of an implementation intervention to increase patient. Age and Ageing. Londres: v. 47 n.1, p. 112-119, 2017. Disponível em: <https://www.ncbi.nlm.nih.gov/pubmed/28985310>. Acesso em: 19 jan. 2018.

MIURA, Shinya et al. Normal Baseline Function Is Associated With Delayed Rehabilitation in Critically III Children. Journal of Intensive Care Medicine, p. 0885066618754507, 2018. Disponível em: <https://www.ncbi.nlm.nih.gov/ pubmed/29357778>. Acesso em: 21 jan. 2018. 
MOTA, C. M.; SILVA, V. G. A segurança da mobilização precoce em pacientes críticos: uma revisão de literatura. Interfaces Científicas-Saúde e Ambiente, Aracaju, v. 1, n. 1, p. 83-91, 2012. Disponível em: < https://periodicos.set.edu.br/index.php/saude/article/download/181/107 >. Acesso em: 23 jan. 2018.

PAIS, S. A. O doente crítico. 2012. Dissertação (Mestrado em Enfermagem, Especialização Em Enfermagem Médico-Cirúrgica) - Instituto de Ciências da Saúde, Universidade Católica Portuguesa, Viseu. Disponível em: <https://repositorio.ucp.pt/handle/10400.14/10219>. Acesso em: 20 jan, 2018.

PERME, C.; CHANDRASHEKAR, R. Early mobility and walking program for patients in intensive care units: creating a standard of care, American Journal Critical Care, v. 18, n. 3, p. 212-221, 2009. Disponível em <https://www.ncbi.nlm.nih.gov/ pubmed/19234100>. Acesso em: 14 jan. 2018.

SARTI, T. C.; VECINA, M. V. A.; FERREIRA, P. S. N. Mobilização precoce em pacientes críticos. J Health Sci Inst. v. 34, n. 3, p. 177-182, 2016. Disponível em: <http://www.scielo. br/pdf/rbti/v24n1/03.pdf>. Acesso em: 23 jan. 2018.

WIECZOREK, B. et al. PICU Up!: Impact of a Quality Improvement Intervention to Promote Early Mobilization in Critically III Children. Pediatric Critical Care Medical, Mount Prospect, v. 17, n. 12, p. e559-e566, 2016. Disponível em: <https://www.ncbi. nlm.nih.gov/pubmed/27759596>. Acesso em: 20 jan. 2018.

Recebido em: 26-04-2018

Aceito em: 01-07-2019 